\title{
A Retrospective Analysis of Pulmonary Cryptococcosis Following Kidney Transplantation: Clinical Presentation, Treatment and Effectiveness
}

\section{Shuyang CHEN}

Fuzhou General Hospital of Fujian Medical University, Dongfang Hospital of Xiamen University, The 900th Hospital of the Joint Logistic Support Force

\section{Meiyan CHEN}

Fuzhou General Hospital of Fujian Medical University, Dongfang Hospital of Xiamen University, The 900th Hospital of the Joint Logistic Support Force

\section{Lei GU}

The First Affiliated Hospital of Chongqing Medical University

\section{Qing WANG}

The Third Affiliated People's Hospital of Fujian University of Traditional Chinese Medicine

\section{Yanjing YOU}

Fuzhou General Hospital of Fujian Medical University, Dongfang Hospital of Xiamen University, The 900th Hospital of the Joint Logistic Support Force

\section{Huijuan WANG}

Fuzhou General Hospital of Fujian Medical University, Dongfang Hospital of Xiamen University, The 900th Hospital of the Joint Logistic Support Force

\section{Guoxiang LAI}

Fuzhou General Hospital of Fujian Medical University, Dongfang Hospital of Xiamen University, The 900th Hospital of the Joint Logistic Support Force

\section{Zongyang YU}

Fuzhou General Hospital of Fujian Medical University, Dongfang Hospital of Xiamen University, The 900th Hospital of the Joint Logistic Support Force

\section{Wen WEN ( $\nabla$ fjfzfisher@163.com )}

Fuzhou General Hospital of Fujian Medical University, Dongfang Hospital of Xiamen University, The 900th Hospital of the Joint Logistic Support Force

\section{Research Article}

Keywords: pulmonary cryptococcosis, organ transplant, therapeutic approach 
DOI: https://doi.org/10.21203/rs.3.rs-986029/v1

License: (c) (1) This work is licensed under a Creative Commons Attribution 4.0 International License. Read Full License 


\section{Title Page}

\section{A Retrospective Analysis of Pulmonary Cryptococcosis Following Kidney Transplantation: Clinical Presentation, Treatment and Effectiveness}

CHEN Shuyang ${ }^{1}$, CHEN Meiyan ${ }^{1}$, GU Lei ${ }^{2}$, WANG Qing ${ }^{3}$, YOU Yanjing ${ }^{1}$, WANG Huijuan

${ }^{1}$, LAI Guoxiang ${ }^{1}$, Yu Zongyang ${ }^{1}$, WEN Wen ${ }^{1 *}$

1.Department of Respiratory and Critical Care Medicine, Fuzhou General Hospital of Fujian Medical University, Dongfang Hospital of Xiamen University, The 900th Hospital of the Joint Logistic Support Force, PLA, Gulou, Fuzhou 350025, China

2. The First Affiliated Hospital of Chongqing Medical University, Chongqing 400016, China

3.The Third Affiliated People's Hospital of Fujian University of Traditional Chinese

Medicine,Fuzhou 350108, China

Keywords: pulmonary cryptococcosis, organ transplant, therapeutic approach.

Suggested Running Title: Pulmonary Cryptococcosis in Organ Recipients

Correspondence Author: Dr. WEN Wen, Email: fjfzfisher@163.com, Phone:

(+86)13805010646, Department of Respiratory and Critical Care Medicine, Fuzhou General Hospital of Fujian Medical University, Dongfang Hospital of Xiamen University, The 900th Hospital of the Joint Logistic Support Force, PLA, Gulou, Fuzhou 350025, China.

Alternate Corresponding Author: Dr. CHEN Shuyang, Email: georgechan_fjmu@163.com, Phone: (+86)13666012245.Department of Respiratory and Critical Care Medicine, Fuzhou General Hospital of Fujian Medical University, Dongfang Hospital of Xiamen University, The 900th Hospital of the Joint Logistic Support Force, PLA, Gulou, Fuzhou 350025, China.

Summary: This article retrospectively reviewed 18 patients with pulmonary cryptococcosis following kidney transplantation, analysed their different clinical presentations, therapeutic approaches and effectiveness, discussed possibilities for early diagnosis and effective treatment for immunosuppressed patients. 


\begin{abstract}
Background: The use of immunosuppressors and a relatively weaken cell-mediated immunity make organ transplant recipients particularly vulnerable to cryptococcosis infection. Patients infected usually present only nonspecific symptoms, making it extremely possible for misdiagnosis and inappropriate choice of therapeutic approach.
\end{abstract}

Methods: We compiled and analysed data of patients received kidney transplant in our hospital between April 2006 to January 2021.

Results: 18 patients were enrolled into the study, ranging between 27-68 years old. The median time from kidney transplantation to pathologically-confirmed infection was 4.09 years. All patient's respiratory system was affected, showing symptoms including sputumproducing cough and fever. 3 patients $(16.67 \%)$ also developed central nervous system (CNS) infections. Nodule-shaped infectious sites were frequently observed $(10,58.82 \%)$ in chest CT. Blood works showed no specific changes. 7 patients received thoracoscopic lobectomy in suspicion of lung cancer. 3 patients first received antifungal therapy for a period of time and then underwent thoracoscopic lobectomy. No recurrence whatsoever was observed in all 10 surgically-intervened patients. 8 patients received only antifungal therapy, 7 of them showed a substantial reduction in the size of the infectious site. Fluconazole was most frequently prescribed for antifungal therapy.

Conclusion: Most patients developed pulmonary cryptococcosis 2 years after transplantation. Patients usually demonstrate symptoms like fever and sputum-producing cough. The possibility of cryptococcal meningitis shouldn't be ruled out if corresponding symptoms occur. CT presentation may be confused with lung cancer. Fluconazole is commonly prescribed for treatment and can usually yield satisfied outcome. In patients received unsatisfactory antifungal therapy, surgical therapy should be considered a possibility. 


\section{Introduction}

Opportunistic infections following solid organ transplantation (SOT) have been considered one of the major contributing factors for mortality and morbidity in organ transplant recipients. The use of immunosuppressors and a relatively weaken cell-mediated immunity make SOT recipients particularly vulnerable to invasive fungal diseases (IFD). Study in United States concluded the incidence rate of cryptococcosis in SOT recipients at 1-2\%, with the 12-month incidence rate at $0.2 \% \cdot{ }^{[1][2]}$ Similar study in China concluded this figure at $0.76 \% \cdot{ }^{[3]}$ The clinical presentations of cryptococcal infections are still not clearly defined, whereas patients infected usually present only nonspecific symptoms or no symptoms at all, making it extremely possible for misdiagnosis and inappropriate choice of therapeutic approach.

For this study, we retrospectively reviewed 18 patients received kidney transplantation in our hospital and later developed Pulmonary Cryptococcosis. These patients received different therapy, ranging from pure surgical removal of infectious site to antifungal therapy combined with surgical intervention. We hope that when closely re-examine the steps we took to diagnose and treat these patients can we better understand this disease, making it possible for physicians to make timely diagnosis and thus provide smooth recovery for future patients.

\section{Methods}

\subsection{Data Collection.}

We conducted this study in the 900th Hospital of Joint Logistic Forces, a regional centre for kidney transplant and respiratory diseases located in Fuzhou, China. Patients with cryptococcosis infection following kidney transplantation (KT) between April 2006 to January 2021 were reviewed and included 18 patients to the study. We compiled and analysed the basic demographic data, out-patients' visiting records, CT images, laboratory results, therapeutic procedures and outcomes.

This study was approved by the Ethic Committee of the 900th Hospital of Joint Logistic Forces.

\subsection{Analysis}

Most variables, including time-related data, laboratory counts and changes in CT images are reported with mean values and standard deviations. Certain descriptive statistics were used to describe basic information of the patients.

All data were analysed using SPSS 26.0 (SPSS Inc., Chicago, IL, USA).

\section{Results}

\subsection{Basic information}

The 18 patients' demographical characteristics are summarized in Table $1(\mathrm{n}=18)$. We define the time between kidney transplantation and pathologically-confirmed pulmonary

cryptococcosis as TTI (Transplant to Infection), which averaged 1645.61 \pm 1044.19 days, roughly $4.51 \pm 2.86$ years.

Table 1 Demographic Data $(n=18)$

\begin{tabular}{|c|l|}
\hline Variable & $\mathbf{N}(\%)$ \\
\hline Gender & \\
\hline Male & $14(77.78 \%)$ \\
\hline Female & $4(22.22 \%)$ \\
\hline
\end{tabular}




\begin{tabular}{|l|l|}
\hline Age of Kidney Transplantation & $41.61 \pm 12.56$ \\
\hline Age of Cryptococcosis Diagnosis & $46.11 \pm 12.15$ \\
\hline $\begin{array}{l}\text { Time between Transplant to } \\
\text { Infection(T2I)/Days }\end{array}$ & $1645.61 \pm 1044.19$ \\
& $(4.51 \pm 2.86$ years $)$ \\
\hline Underlying Diseases & \\
\hline Hypertension & $6(33.33 \%)$ \\
\hline Type 2 Diabetes Mellitus & $4(22.22 \%)$ \\
\hline Type B Viral Hepatitis & $4(22.22 \%)$ \\
\hline Coronary Heart Disease & $1(5.56 \%)$ \\
\hline Immunosuppressive Regimen & \\
\hline Tacrolimus (FK-506) & $9(50.00 \%)$ \\
\hline Cyclosporin A & $1(5.56 \%)$ \\
\hline Mycophenolate mofetil (MMF) & $7(38.89 \%)$ \\
\hline Mizoribine & $1(5.56 \%)$ \\
\hline Methylprednisolone & $4(22.22 \%)$ \\
\hline Prednisone & $4(22.22 \%)$ \\
\hline Organs Affected & $18(100.00 \%)$ \\
\hline Lungs & $3(16.67 \%)$ \\
\hline Central Nervous System & \\
\hline
\end{tabular}

In terms of underlying diseases, the most common one was Hypertension $(6,33.33 \%)$, other diseases involved T2DM (4, 22.22\%), Type B Hepatitis (4, 22.22\%). No patient was positive for HIV.

All patients routinely accepted immunosuppressive treatments following kidney transplantation, regimens used for these patients are listed below. It's worth noticing that data here only recorded patients receiving drugs from our hospital, some patients may receive immunosuppressive regimens from local clinics for health insurances concerns.

\subsection{Imaging Findings}

We were able to retrieve 17 patients' chest CT images both at and after infection. 1 patient, however, only has biopsy studies available. 2 cases with central nervous system infected have MR brain images available (Figure 1). Chest CT presentations and characteristics are summarized in Table 2. Infections were usually presented in shapes of nodules (10,58.82\%), imaging changes were most commonly observed in the middle lobe of right lung. Measurements of all nodules-shaped infection averages $2.04 \times 1.49 \mathrm{~cm}$ in diameters. Figure 24 demonstrated some classical changes observed in chest CT images. With regards to particular imaging changes, we were able to discover spiculation in 12 cases, amounting to $70.59 \%$ of all cases.

Figure 1 MR Image with cryptococcal infection, demyelination pointed with arrows 


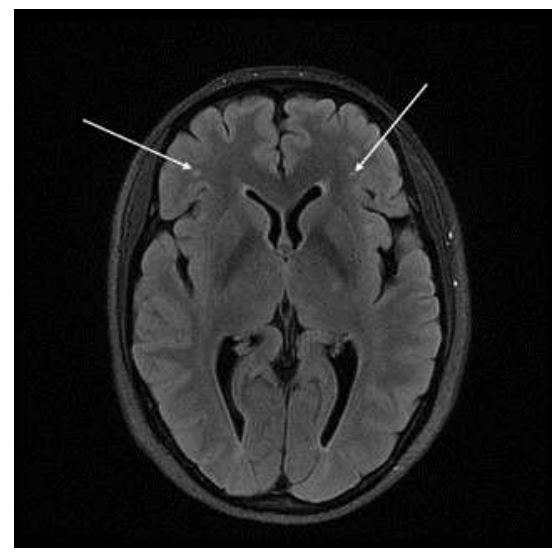

Figure 2 Nodule shaped infectious site

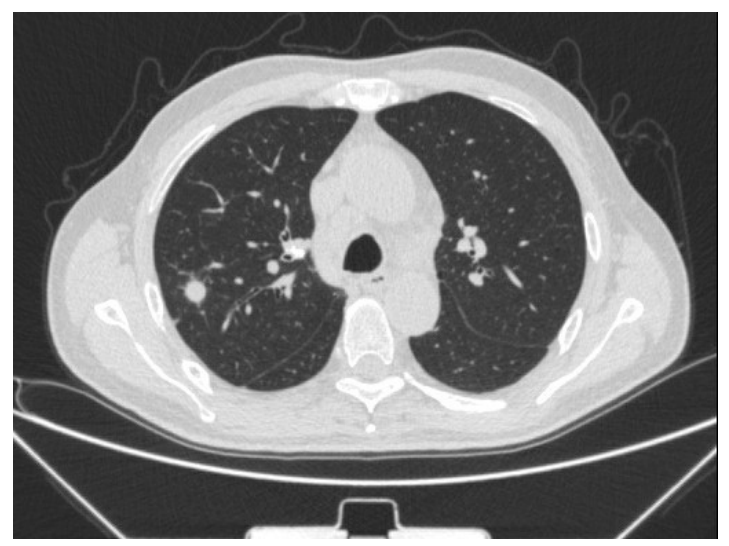

Figure 3 Cavity formed within the nodule-shaped infectious site

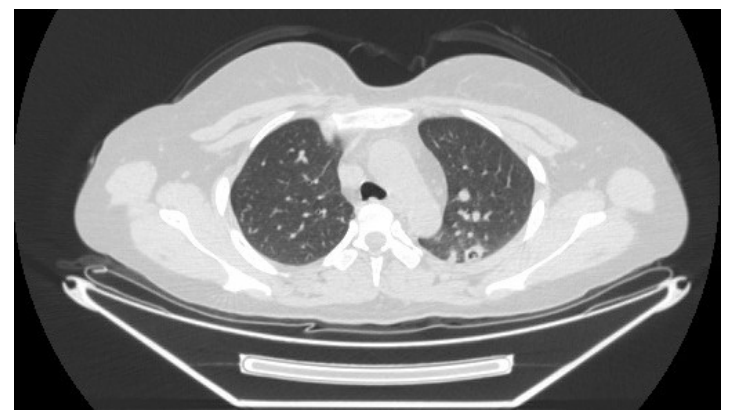

Figure 4 Multiple mass and nodule-shaped infectious site

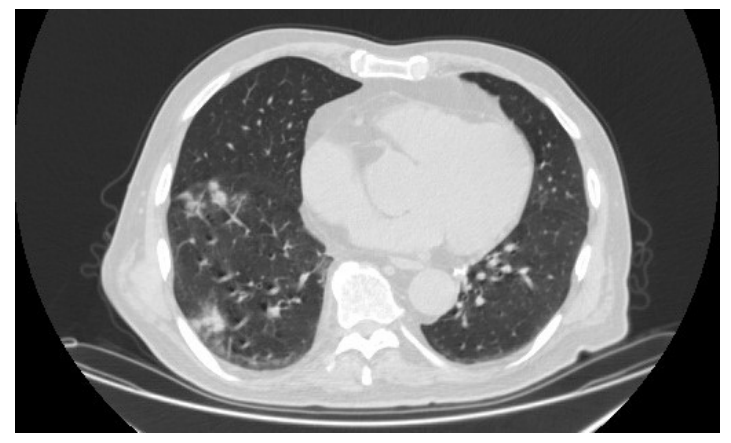

Table 2 Imaging Presentations and Characteristics $n=17$ ( 1 case's $C T$ images could not be retrieved)

\section{Characteristics}

$\mathbf{N}(\%)$ 


\begin{tabular}{|l|l|}
\hline Nodules & $10(58.82 \%)$ \\
\hline Masses & $2(11.76 \%)$ \\
\hline Nodules and Masses & $5(29.41 \%)$ \\
\hline Lobes Infected & \\
\hline R, Superior Lobe & $4(23.53 \%)$ \\
\hline R, Middle Lobe & $9(52.94 \%)$ \\
\hline R, Inferior Lobe & $2(11.76 \%)$ \\
\hline L, Superior Lobe & $3(17.65 \%)$ \\
\hline L, Inferior Lobe & $6(35.29 \%)$ \\
\hline Diameter & $(2.04 \pm 0.74) \times(1.49 \pm 0.50)$ \\
\hline Imaging Presentations & \\
\hline Spiculation & $12(70.59 \%)$ \\
\hline Pleural Indentation & $3(17.65 \%)$ \\
\hline Lobulation & $3(17.65 \%)$ \\
\hline Infiltration & $7(41.18 \%)$ \\
\hline Cavity & $6(35.29 \%)$ \\
\hline Halo Sign & $1(5.88 \%)$ \\
\hline Angio-Assemblage Sign & $1(5.88 \%)$ \\
\hline 3.3 Laboratory Findings & \\
\hline
\end{tabular}

\subsection{Laboratory Findings}

Blood routine tests were not able to determine abnormal changes for this group of patients. Upon infection, the white blood cells count (WBC) was $8,682 \pm 1,793$ cells per mm3. CRP averages $9.41 \pm 8.12$ in 11 cases. In 12 cases received follow-up checks 3 months after diagnosis, WBC averages $8322 \pm 2441$ cells per mm3. At 6 months' interval, WBC averages $7,267 \pm 2,418$ cells per mm3 in 7 cases. At 9 months' interval, WBC averages $8,174 \pm 1495$ cells per mm3 in 9 cases. Showing no particular abnormality. Other blood works and blood biochemistry tests data were compiled in Table 3-1 and Table 3-2.

Table 3-1 Blood Works in Different Time Intervals

\begin{tabular}{|l|l|l|l|l|}
\hline & $\begin{array}{l}\text { Infection } \\
\mathbf{n}=\mathbf{1 6}\end{array}$ & $\begin{array}{l}\mathbf{3} \text { Months } \\
\mathbf{n}=\mathbf{1 2}\end{array}$ & $\begin{array}{l}\mathbf{6} \text { Months } \\
\mathbf{n = 7}\end{array}$ & $\begin{array}{l}\mathbf{9} \text { Months } \\
\mathbf{n}=\mathbf{9}\end{array}$ \\
\hline Neu\# & $6,067 \pm 1,632$ & $5,483 \pm 2,280$ & $4,624 \pm 1,737$ & $5,520 \pm 1,277$ \\
\hline Neu\% & $68.7250 \pm 8.596$ & $64.3617 \pm 8.278$ & $62.9057 \pm 4.091$ & $67.0711 \pm 6.323$ \\
\hline Lymph\# & $1.8894 \pm 0.6695$ & $2.0375 \pm 0.5402$ & $1.9171 \pm 0.5428$ & $1.8778 \pm 0.4053$ \\
\hline Lymph\% & $21.8763 \pm 7.3764$ & $25.7283 \pm 6.8503$ & $27.0771 \pm 3.7794$ & $23.4044 \pm 5.6691$ \\
\hline Mono\% & $6.7537 \pm 1.3399$ & $7.3367 \pm 2.0281$ & $7.2771 \pm 1.6028$ & $7.0600 \pm 1.1541$ \\
\hline Eo\% & $1.1263 \pm 0.7257$ & $1.5033 \pm 1.3146$ & $1.6200 \pm 1.1527$ & $1.0933 \pm 0.6016$ \\
\hline
\end{tabular}




\begin{tabular}{|l|l|l|l|l|}
\hline Baso\% & $0.2806 \pm 0.2329$ & $0.2200 \pm 0.1632$ & $0.1629 \pm 0.1426$ & $0.2822 \pm 0.2527$ \\
\hline Hemo\# & $137.8750 \pm 18.8640$ & $138.6667 \pm 19.3501$ & $141.1429 \pm 20.1943$ & $145.0000 \pm 19.5192$ \\
\hline RBC\# & $4.7219 \pm 0.6106$ & $4.9258 \pm 0.7385$ & $4.7529 \pm 0.5001$ & $4.8489 \pm 0.4813$ \\
\hline HCT & $41.9625 \pm 4.7986$ & $41.6917 \pm 5.5685$ & $41.6571 \pm 5.8785$ & $42.4556 \pm 5.0202$ \\
\hline PLT\# & $182.1875 \pm 45.5093$ & $183.9167 \pm 32.7788$ & $171.0000 \pm 22.5758$ & $182.3333 \pm 19.8809$ \\
\hline
\end{tabular}

Table 3-2 Blood Biochemistry Tests in Different Time Intervals

(*1 patient experienced Transplant Kidney Failure when infected, thus was not taken into analysis)

\begin{tabular}{|l|l|l|l|l|}
\hline & Infection & 3 Months & 6 Months & 9 Months \\
\hline \multirow{2}{*}{ ALT } & $12.5400+4.9073$ & $\begin{array}{l}15.5556+8.1832 \\
(\mathrm{n}=9)\end{array}$ & $\begin{array}{l}15.4250+7.4567 \\
(\mathrm{n}=8)\end{array}$ & $\begin{array}{l}17.7750+10.0972 \\
(\mathrm{n}=8)\end{array}$ \\
\hline \multirow{2}{*}{ AST } & $14.6733+4.2137$ & $\begin{array}{l}18.5800+6.1994 \\
(\mathrm{n}=5)\end{array}$ & $\begin{array}{l}14.1500+2.9218 \\
(\mathrm{n}=4)\end{array}$ & $\begin{array}{l}17.5400+7.2879 \\
(\mathrm{n}=5)\end{array}$ \\
\hline \multirow{2}{*}{ TBIL } & $\begin{array}{l}9.9600+5.5854 \\
(\mathrm{n}=15)\end{array}$ & $\begin{array}{l}7.4500+3.9975 \\
(\mathrm{n}=8)\end{array}$ & $\begin{array}{l}7.5571+4.8699 \\
(\mathrm{n}=7)\end{array}$ & $\begin{array}{l}12.7333+6.9778 \\
(\mathrm{n}=9)\end{array}$ \\
\hline \multirow{2}{*}{ BUN } & $\begin{array}{l}6.5800+2.5705 \\
(\mathrm{n}=15)\end{array}$ & $\begin{array}{l}7.4667+2.9921 \\
(\mathrm{n}=9)\end{array}$ & $\begin{array}{l}6.7429+2.3487 \\
(\mathrm{n}=7)\end{array}$ & $\begin{array}{l}6.8667+3.6753 \\
(\mathrm{n}=9)\end{array}$ \\
\hline \multirow{2}{*}{ Cr* } & $\begin{array}{l}9.9786+21.9284 \\
(\mathrm{n}=14)\end{array}$ & $\begin{array}{l}89.4000+41.5377 \\
(\mathrm{n}=8)\end{array}$ & $\begin{array}{l}91.7833+27.1780 \\
(\mathrm{n}=6)\end{array}$ & $\begin{array}{l}99.2000+24.4594 \\
(\mathrm{n}=8)\end{array}$ \\
\hline
\end{tabular}

Most cases studied here were originally treated in thoracic surgery department, where a strict cryptococcosis test protocol was not followed, leading to only 2 patients tested for serum cryptococcal antigen and yielded positive results. The remaining 16 patients did not receive cryptococcosis capsular antigen tests.

In 3 cases with CNS cryptococcosis infections, cryptococcal capsular antigen tested positive in cerebrospinal fluid.

Immunology test results were compiled in Table 3-3. Comparison could not be drawn as only 2 patients tested again at 3 months interval.

Table 3-3 FCM Blood Antibodies Tests Upon Infection

\begin{tabular}{|l|l|}
\hline Index & Results(n=8) \\
\hline CD3 & $77.9375 \pm 5.8559$ \\
\hline CD4 & $41.0375 \pm 10.7420$ \\
\hline CD8 & $34.2875 \pm 7.6488$ \\
\hline CD4/CD8 & $1.2875 \pm 0.5111$ \\
\hline
\end{tabular}

All patients were diagnosed using lung biopsy combined with PAS\&PAMM stain.

\subsection{Clinical Manifestations}

The most commonly affected organ was lung $(18,100 \%)$, followed by 3 cases with central nervous system infected. Cryptococcal meningitis (CM) was identified in said 3 patients, showing symptoms of headache and respiratory symptoms of cough with yellow sputum. 1 $\mathrm{CM}$ patient exhibited more severe CNS symptoms involving vomiting, head-spinning and fever. 3 cases $(16.67 \%)$ showed no symptoms whatsoever and was identified during a routine 
chest CT exam, while the remaining 15 patients (83.33\%) experienced one or more kinds of symptoms at least 30 days before diagnosis. Most patients presented respiratory symptoms like cough $(10,55.56 \%)$ with sputum $(9,50.00 \%)$. Clinical manifestations analyzed and compiled to Table 4.

Table 4 Clinical Manifestations

\begin{tabular}{|l|l|}
\hline Symptoms & Results(n=18) \\
\hline Cough & $10(55.56 \%)$ \\
\hline Sputum & $9(50.00 \%)$ \\
\hline Yellow & $3(16.67 \%)$ \\
\hline White & $6(33.33 \%)$ \\
\hline Shortness of Breath & $1(5.56 \%)$ \\
\hline Fever & $3(16.67 \%)$ \\
\hline Pharyngalgia & $3(16.67 \%)$ \\
\hline Headache & $2(11.11 \%)$ \\
\hline Vomiting & $1(5.56 \%)$ \\
\hline
\end{tabular}

\subsection{Treatments and Outcomes}

The opportunistic infections after organ transplantation were not seriously monitored for a period of time, considering post-transplant patients usually make follow-up checks in surgical units, most of the cryptococcal infections were removed surgically rather than guidelines today suggested. In this cohort of study, 10 patients $(55.56 \%)$ received thoracoscopic lobectomy. It's worth noticing that 3 patients received surgery after antifungal intervention (both intravenous and oral) proved unsatisfactory. A few patients $(2,11.11 \%)$ received lobectomy later developed pleural effusion and post operation infections, typically presented with pleural effusion, as demonstrated in Figure 5-6.

Figure 5 Pleural effusion observed in left side of the chest

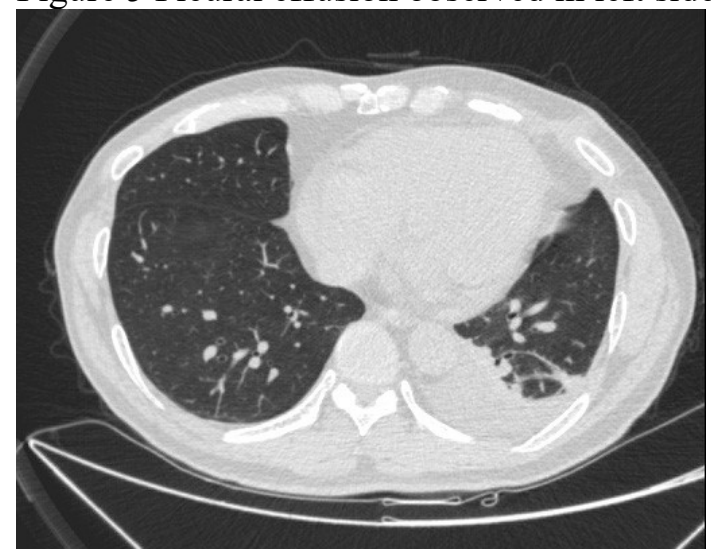

Figure 6 Pleural effusion observed in right side of the chest 


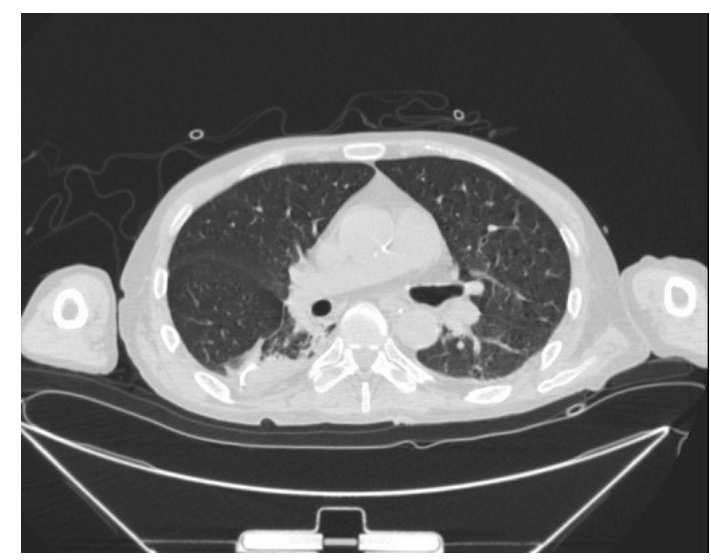

Here, we divide our 18 patients into 3 categories: Surgical group (patients receiving surgery removal with or without continuous antifungal therapy), Antifungal + Surgical group (patients received unsatisfactory antifungal therapy prior to surgical procedure), Antifungal group (patients received only antifungal therapy). Details regarding the approaches taken are listed in Table 5.

\section{Table 5 Therapeutic Approaches}

$N D$ : No Data (patients receiving drugs somewhere else); $F C Z$ : fluconazole; $V C Z$ : voriconazole; $A m B$ : amphotericin B; Drugs mostly given orally unless specified otherwise.

\begin{tabular}{|c|c|c|c|c|}
\hline Group & \multicolumn{2}{|c|}{ Case } & \multicolumn{2}{|c|}{ Post-Operation Therapy } \\
\hline \multirow{7}{*}{ Surgical } & & 1 & \multicolumn{2}{|l|}{ Not Used } \\
\hline & \multicolumn{2}{|r|}{2} & \multicolumn{2}{|c|}{$\mathrm{FCZ}($ ivgtt $) 400 \mathrm{mg} /$ day $\times 6$ weeks } \\
\hline & \multicolumn{2}{|r|}{3} & \multicolumn{2}{|c|}{ FCZ $200 \mathrm{mg} /$ day $\times 16$ weeks } \\
\hline & \multicolumn{2}{|r|}{5} & \multicolumn{2}{|l|}{ Not Used } \\
\hline & \multicolumn{2}{|r|}{6} & \multicolumn{2}{|c|}{ VCZ 400mg/day (Duration Unknown) } \\
\hline & & 8 & \multicolumn{2}{|c|}{ FCZ $400 \mathrm{mg} /$ day $\times 8$ weeks } \\
\hline & \multicolumn{2}{|r|}{18} & \multicolumn{2}{|c|}{$\begin{array}{l}\mathrm{FCZ}(\text { ivgtt }) 400 \mathrm{mg} / \text { day } \times 2 \text { weeks } \\
\mathrm{FCZ} 200 \mathrm{mg} / \text { day } \times 12 \text { weeks }\end{array}$} \\
\hline Group & Case & Pre- & Operation Therapy & Post-Operation Therapy \\
\hline \multirow{5}{*}{$\begin{array}{c}\text { Antifungal } \\
+ \\
\text { Surgical }\end{array}$} & 7 & \multicolumn{2}{|c|}{$\mathrm{ND} \times 1$ Year } & Not Used \\
\hline & 9 & \multicolumn{2}{|c|}{$\begin{array}{l}\text { FCZ }(\text { ivgtt }) 200 \mathrm{mg} / \text { day } \times 3 \\
\text { weeks } \\
\text { FCZ } 200 \mathrm{mg} / \text { day } \times 4 \text { weeks }\end{array}$} & Not Used \\
\hline & 14 & \multicolumn{2}{|c|}{$\begin{array}{l}\text { VCZ }(\text { ivgtt }) 200 \mathrm{mg} / \text { day } \times 4 \\
\text { weeks } \\
\text { FCZ } 200 \mathrm{mg} / \text { day } \times 1 \text { week }\end{array}$} & $\mathrm{FCZ} 200 \mathrm{mg} /$ day $\times 32$ weeks \\
\hline & Case & & Induction Therapy & Consolidation Therapy \\
\hline & 4 & & $\begin{array}{r}\text { Z(ivgtt) } 400 \mathrm{mg} / \text { day } \times 3 \\
\text { weeks }\end{array}$ & $\begin{array}{l}\text { FCZ 400mg/day (Duration } \\
\text { Unknown) }\end{array}$ \\
\hline
\end{tabular}




\begin{tabular}{|c|c|c|}
\hline 10 & FCZ $200 \mathrm{mg} /$ day $\times 1$ week & FCZ 200mg/day $\times 6$ weeks \\
\hline 11 & FCZ (Dosage Unknown) & $\begin{array}{l}\mathrm{FCZ} \times 48 \text { weeks (Dosage } \\
\text { Unknown) }\end{array}$ \\
\hline 12 & $\begin{array}{r}\text { FCZ(ivgtt) } 350 \mathrm{mg} / \text { day } \times 2 \\
\text { days }\end{array}$ & $\begin{array}{l}\text { FCZ 400mg/day (Duration } \\
\text { Unknown) }\end{array}$ \\
\hline 13 & $\begin{array}{r}\text { FCZ(ivgtt) } 400 \mathrm{mg} / \text { day } \times 3 \\
\text { days }\end{array}$ & $\begin{array}{l}\text { FCZ 400mg/day (Duration } \\
\text { Unknown) }\end{array}$ \\
\hline 15 & $\begin{array}{r}\text { VCZ 200mg/day (Duration } \\
\text { Unknown) }\end{array}$ & FCZ $200 \mathrm{mg} /$ day $\times 40$ weeks \\
\hline 16 & $\begin{array}{r}\text { FCZ 200mg/day } \times 3 \text { days } \\
\text { VCZ(ivgtt) } 200 \mathrm{mg} / \text { day } \times 3 \\
\text { days }\end{array}$ & VCZ $200 \mathrm{mg} /$ day $\times 8$ weeks \\
\hline 17 & $\begin{array}{r}\text { FCZ(ivgtt) } 400 \mathrm{mg} / \text { day } \times 5 \\
\text { days } \\
\text { AmB(iv) } 5 \mathrm{mg} / \text { day } \times 5 \text { days } \\
\end{array}$ & ND \\
\hline
\end{tabular}

A closer look at all the patients' antifungal regimen can shed lights on their corresponding outcomes. Guidelines published in 2010 suggest that fluconazole maintenance therapy should be continued for at least 6-12 months, very few patients in our study exceed that suggested duration. ${ }^{[17]}$ It's also recommended, however, for patients underwent surgery to also subsequently receive antifungal therapy for a period of time, which was also not followed in our patients.

As no patients underwent surgical procedures showed diagnostically meaningful recurred pulmonary infection, we focus on the changes in chest $\mathrm{CT}$ images of patients in antifungal group. We define the first abnormal chest $\mathrm{CT}$ result as the starting point and continuing observe and measure the size of the infectious site in patients receiving antifungal therapy. If a patient exhibited more than one sites of infection, we select the largest nodule-shaped infectious site for continuous measurement (Table 6).

Table 6 Changes in size of nodule-shaped infection ( $\mathrm{mm}$ )

$N D$ : No Data;

\begin{tabular}{|l|l|l|l|l|l|l|l|l|}
\hline No. & Infected & $\mathbf{1}$ Month & $\mathbf{2}$ Months & $\mathbf{3}$ Months & $\mathbf{6}$ Months & $\mathbf{9}$ Months & $\mathbf{1}$ Year & $\mathbf{2}$ Years \\
\hline 4 & $30.27 \times 22.71$ & $\mathrm{ND}$ & $\mathrm{ND}$ & $19.88 \times 14.05$ & $\mathrm{ND}$ & $9.20 \times 7.37$ & $3.18 \times 2.12$ & ND \\
\hline 10 & $23.01 \times 13.56$ & $\mathrm{ND}$ & $13.58 \times 13.23$ & $\mathrm{ND}$ & $\mathrm{ND}$ & $\mathrm{ND}$ & $\mathrm{ND}$ & $\mathrm{ND}$ \\
\hline 11 & $9.14 \times 8.71$ & $9.06 \times 8.67$ & $10.83 \times 10.39$ & $\mathrm{ND}$ & $15.72 \times 13.79$ & $\mathrm{ND}$ & $12.12 \times 10.7$ & $13.19 \times 13.06$ \\
\hline 12 & $18.02 \times 17.22$ & $15.09 \times 13.79$ & $11.46 \times 11.41$ & $\mathrm{ND}$ & $\mathrm{ND}$ & $12.34 \times 10.08$ & $11.18 \times 9.79$ & ND \\
\hline 13 & $33.74 \times 25.09$ & $\mathrm{ND}$ & $\mathrm{ND}$ & $21.11 \times 16.79$ & $\mathrm{ND}$ & $\mathrm{ND}$ & $\mathrm{ND}$ & $\mathrm{ND}$ \\
\hline 15 & $13.90 \times 10.04$ & $12.02 \times 8.94$ & $11.75 \times 9.28$ & $\mathrm{ND}$ & $9.52 \times 7.16$ & $9.48 \times 6.64$ & $9.97 \times 7.28$ & ND \\
\hline 16 & $10.62 \times 8.19$ & $11.25 \times 10.08$ & $\mathrm{ND}$ & $7.30 \times 5.90$ & $5.94 \times 5.18$ & ND & ND & $5.62 \times 5.07$ \\
\hline 17 & $10.65 \times 8.88$ & $14.26 \times 10.14$ & $\mathrm{ND}$ & $\mathrm{ND}$ & $\mathrm{ND}$ & $\mathrm{ND}$ & $\mathrm{ND}$ & $\mathrm{ND}$ \\
\hline
\end{tabular}

In Figure 7, a tendency of continuous reduction in the size of the infections can be observed, most prominently in the first 3 months of antifungal therapy. However, an uptick can be seen in patient 11 , despite the continuing application of antifungal therapy, this patient's infection 
site grew to $165.16 \%$ compared with original infection 6 months into antifungal therapy, and dropped to $147.13 \% 2$ years after infected. In patients revisited at 1 year and 2 years interval after infected, the size of the infection is mostly consistent. Considering there may be errors and inaccuracy in measuring the size of the infection, as well as the limited number of patients making follow-up visits in this cohort, we decided not to further calculate exactly when the measurements drop most significantly.

Figure 7 Changes of diameters in different time intervals (percentage)

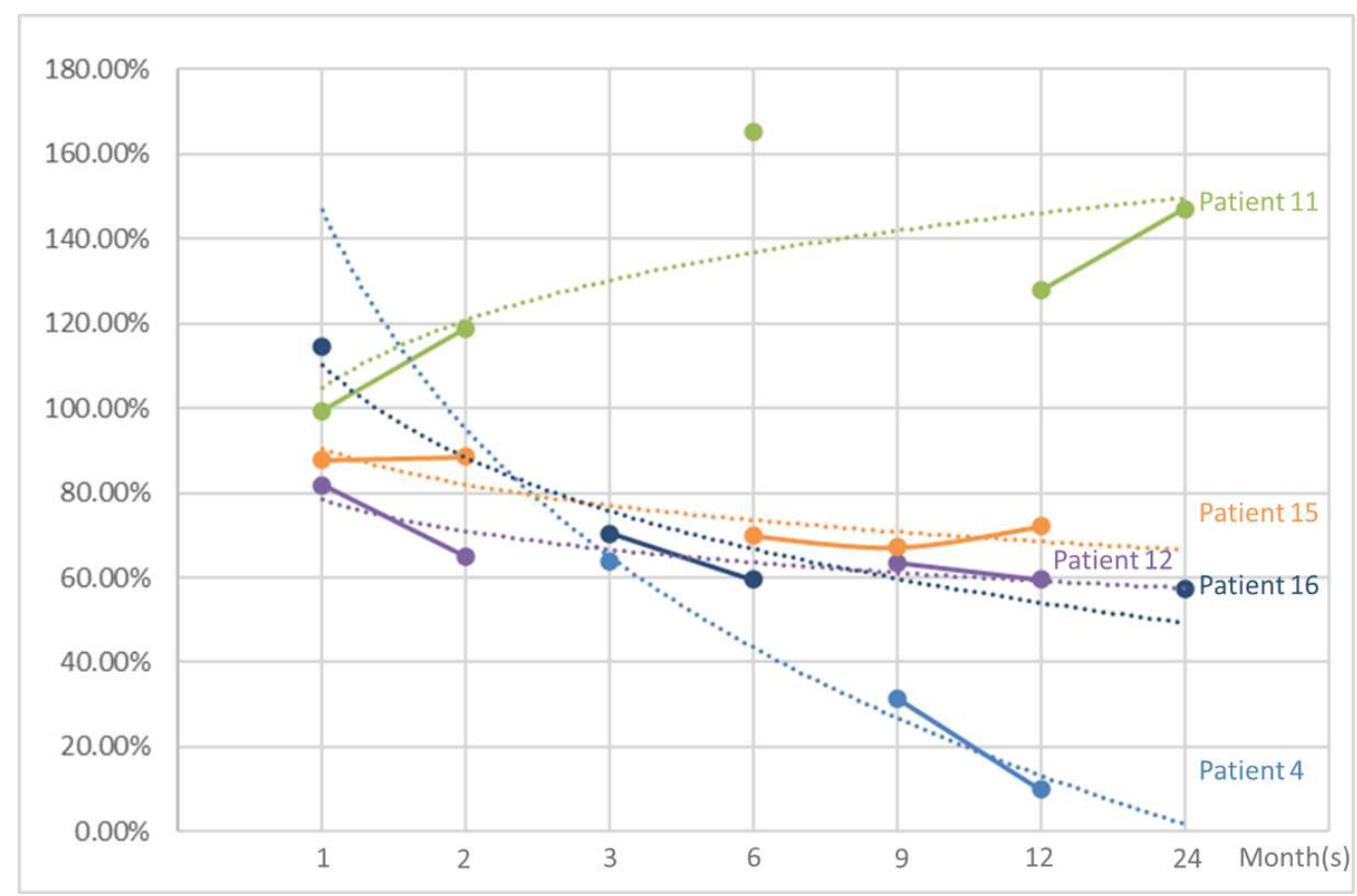

\section{Discussion}

Consistent with most of the researches on this field, cryptococcal infection following kidney transplant is a late occurring one, with most of our patients $(13,72.22 \%)$ infected 2 years after transplantation. In a retrospective analysis included patients over the past 30 years, the occurring time was $5.16 \pm 3.97$ years. Considering the number of included patients in our study is significantly smaller, the occurring time $(4.51 \pm 2.86$ years $)$ we calculated is in agreement with the said one ${ }^{[4][5]}$ The occurring time, or defined as TTI time, may give clues to the origin of the pathogen responsible for the infection. A latent infection may be reactivated and leads to late occurred infection, while an early infection may indicate donorsourced infection. Suggesting donor-derived infection should be considered a possibility when encountered patients showing symptoms within the first few months after transplantation. ${ }^{[6]}$

The most common underlying diseases in this group of patients is hypertension $(5,24.91 \%)$, while Type B hepatitis $(4,23.53 \%)$ and Type 2 diabetes mellitus $(4,23.53 \%)$ are also quite common. This is consistent with previous large-scale retrospective analysis. ${ }^{[3]}$ It's also worth noticing that the KT patients are a group of patients with relatively higher risks of developing diabetes mellitus (DM), a study revealed DM patients have higher possibilities of CNS cryptococcal infection and higher mortality rates. ${ }^{[7]}$ Proper management of patients' underlying diseases is of great significance to avoid such infection. Pigeons are discovered as the natural host of certain subtypes of cryptococcosis, ${ }^{[8]}$ when took a closer look at this group of patients' medical files, we found no clear documentation stating or denying whether they had contact with pigeons or live around their habitat. 
Symptomatic infections in this study usually presented symptoms like sputum-producing cough $(9,52.94 \%)$. This non-specific symptom may very well make patients believe that they are suffering from simple flu or cold. The remaining asymptomatic infections were discovered when receiving routine chest $\mathrm{CT}$ exam. These insidious onset presentations emphasize the necessity for more frequent body check-up for SOT recipients. 3 patients with CNS infections presented far more severe symptoms, including fever, headache and vomiting. However, unnecessarily frequent $\mathrm{CT}$ exam expose patients to the harm of radioactive ways. Upon reviewing current articles, we found no clear guidelines or suggestions for a suitable time interval for patients to receive routine CT exam. This practice varies in different hospital and subject to decisions of the doctors.

A study in Taiwan concluded kidney transplant recipients had an elevated risk for cryptococcal infection involving CNS ${ }^{[9]} \mathrm{CNS}$ related cryptococcal infection can be far more severe, cases reported elsewhere include patients present symptoms like seizures, altered mental status and can be fatal despite aggressive therapy. ${ }^{[10]}$

Laboratory exams and chest $\mathrm{CT}$ are the usual approaches to further examine a suspicious cryptococcal infection, yet the selection of exactly which test to perform may vary from doctors to doctors. We compiled a series of blood works results in Table 3-1 and its tendency following diagnosis for a period of time, yet most of the results stay within the normal range and presented no abnormal changes. Methods target more specifically on the pathogen, including culture, direct microscopic, histopathology, serology tests and molecular detection are considered more accurate and are recommended by multiple guidelines. ${ }^{[1]}$ Most of our patients underwent surgical procedures were diagnosed after pathological exams. In patients receiving CT-guided percutaneous needle biopsy (PCNB), histopathological periodicacidSchiff (PAS) stain was used for diagnosis. The importance of positive serum CrAg test is being increasingly stressed on, yet only 3 patients in this study was checked and proved positive. It's worth noticing that the capsular polysaccharides and other antigen detection tests are not routinely performed in most of the hospital laboratory and requires third-party laboratory to carry out the tests. In cases with CNS infection, lumbar puncture is usually performed to identify the responsible pathogen. In a study targeted more directly at the role of serum cryptococcal antigen in the diagnosis of such diseases, it was found that patients with extrapulmonary cryptococcal infection are more likely to be tested positive for serum antigen. ${ }^{[11]}$ The measurement of cerebrospinal fluid (CSF) pressure and white cell counts are also of great importance as these may indicate inflammatory responses. ${ }^{[12]}$ Furthermore, fluorescence-activated cell sorting (FCM) technique has been applied in the diagnosis of cryptococcosis infection. Despite only a few patients in this study received FCM CD4/8 test and yield no specific results, previous study indicates a higher CD4 counts may indicate an asymptomatic patient.

Abundant chest CT images in our study cast lights on how the infection develop and contained. The most common radiographical changes observed here is nodule $(10,58.82 \%)$. There were also changes like masses and even masses associated with nodules. Noduleshaped infections were measured and concluded that the average size is $2.04 \times 1.49 \mathrm{~cm}$. Research on the differences between immunocompetent cryptococcal infection and immunodeficient (including HIV patients) indicated that the immunodeficient patients may demonstrate more abnormal imaging changes, including ground glass opacity (GGO) and halo signs. ${ }^{[13][14]}$ Our patients, however, most commonly presented spiculation around the nodule-shaped infections, halo sign can be observed in one patient. Studies focused on the CT characteristics of pulmonary cryptococcosis indicate that older patients have a tendency of showing GGOs in peripheral fields of the lower lobes. ${ }^{[15]}$

The most notable characteristics in the study is the use of surgical removal as therapeutic plans in cryptococcosis infection. In the guideline published by Infectious Disease Society of America (IDSA) in 2010, surgical approach was only recommended for patients showing persistent radiographic abnormalities and symptoms nonresponding to antifungal therapy. ${ }^{[13]}$ 
Another guideline published by China Medical Society on the diagnosis and treatment of SOT recipients' invasive fungal infection in 2019 also recommend antifungal therapy as primary therapeutic approach. ${ }^{[16][17]}$ Considering the radiographic abnormalities in cryptococcal infections do not bear particular differences than other nodule-shaped pulmonary diseases (eg. lung cancer), surgical procedure usually taken as a result of misdiagnosis. An upside for this approach is obviously the relatively complete removal of the infection. However, the possibilities of post-operation pleural effusion and long-term organizing pneumonia are not ignorable.

In our study, only 1 of all 18 patients received only surgery and no antifungal therapy. As discussed before, surgical removal is not a primarily recommended therapeutic approach in cryptococcosis infection. Fluconazole ( $400 \mathrm{mg} /$ day orally for $6-12$ months) is recommended by both IDSA and Chinese Medical Society guidelines. ${ }^{[18][19]}$ It's clear that basically all our patients did not receive suggested duration of antifungal therapy, except for 1 patient (patient no.14), who not only underwent surgery but also received 32 weeks ( 8 months) of antifungal therapy. In other patients received antifungal therapy, however, only very few patients can follow through recommended duration of therapy. More surprisingly, 3 patients in this study exhibited a growing tendency in the size of the infection, particularly in patient 11 as discussed before. Despite patient 16's infection site started to reduce in size 3 months into the therapy, the overall effectiveness of antifungal therapy in this group of patients is still unsatisfactory. Previous physicians observed the dynamic changes in pulmonary cryptococcal infection and concluded from chest CT that the size of the infection sites usually reduced $67.9 \% 6$ months into antifungal therapy. ${ }^{[20]}$

IDSA guidelines also suggest sequential or step-wise reduction of immunosuppressants, suggesting the dose of corticosteroid be lowered first. While further clinical studies should be performed to further validate the effectiveness of this suggestions, it is of great necessity to determine whether immunosuppressants influence the pharmaceutical effects of the antifungal therapy. ${ }^{[18]}$

Invasive fungal diseases remain one of the most possible post-transplant diseases in SOT recipients. IFD varies in symptoms and lack specific clinical presentations, requiring physicians to be particularly vigilant when immunocompromised or immunosuppressed patients develop respiratory disease-related symptoms, including sputum producing cough and fever. Evidence-based research continuously provide new insights into the treatment and management of these patients. We hope that future study may include more patients received recommended therapy and willing to make more frequent follow-up visits, making it possible for effective and timely adjustment of immunosuppressive regimen and antifungal therapy, improving the quality of life for all patients.

\section{Declarations}

Ethics approval and consent to participate: This study was approved by the Ethical Committee of the $900^{\text {th }}$ Hospital of the Joint Logistics Support Forces, PLA. All patients understood the purpose of this study and gave informed consent to the use of their medical data. This study was carried out strictly in accordance with the Declaration of Helsinki.

Consent for publication: All authors read and approved the publication of this article.

Availability of data and materials: The data-sets used and/or analysed during the current study available from the corresponding author on reasonable request.

Competing interests: All authors declare no conflict of interests.

Funding: This study received no funding.

Authors' information (optional)Author contributions. CHEN S., CHEN M., GU L., WANG Q. contributed equally to this work.CHEN S., CHEN M., GU L., WANG Q 
participated in manuscript drafting.YOU Y, WANG H, LAI G, Yu Z collected and analyzed clinical data. WW participated in data interpretation and manuscript revision.

Acknowledgements. CHEN S. thank Dr. JIN Hongjuan for her guidance and suggestion in the composing of this article. 


\section{References}

[1]Pappas, Peter G. "Cryptococcal infections in non-HIV-infected patients." Transactions of the American Clinical and Climatological Association 124 (2013): 61.

[2]Invasive fungal infections among organ transplant recipients: results of the TransplantAssociated Infection Surveillance Network (TRANSNET)..

[3] Yuchong, Chen, et al. "Cryptococcosis in China (1985-2010): review of cases from Chinese database." Mycopathologia 173.5 (2012): 329-335.

[4]Yang, Ya-li, et al. "Cryptococcosis in kidney transplant recipients in a Chinese university hospital and a review of published cases." International Journal of Infectious Diseases 26 (2014): 154-161.

[5]Zhang Y, Li N, Zhang Y, et al. Clinical analysis of 76 patients pathologically diagnosed with pulmonary cryptococcosis [published correction appears in Eur Respir J. 2013 Jan;41(1):252]. Eur Respir J. 2012;40(5):1191-1200. doi:10.1183/09031936.00168011

[6]Camargo, Jose F., et al. "A cluster of donor-derived C ryptococcus neoformans infection affecting lung, liver, and kidney transplant recipients: Case report and review of literature." Transplant Infectious Disease 20.2 (2018): e12836.

[7]Li, Yingfang, et al. "Cryptococcosis in patients with diabetes mellitus II in mainland China: 1993-2015." Mycoses 60.11 (2017): 706-713.

[8]Mitchell, Thomas G., et al. "Environmental niches for Cryptococcus neoformans and Cryptococcus gattii." Cryptococcus: from human pathogen to model yeast (2010): 235-259.

[9]Chen, Yung-Tai, et al. "Incidence of and risk factors for cryptococcosis in kidney transplant recipients in Taiwan-A nationwide population-based study." International Journal of Infectious Diseases 88 (2019): 154-158.

[10] Franco-Paredes C, Womack T, Bohlmeyer T, et al. Management of Cryptococcus gattii meningoencephalitis. Lancet Infect Dis. 2015;15(3):348-355. doi:10.1016/S14733099(14)70945-4

[11] Singh N, Alexander BD, Lortholary O, et al. Pulmonary cryptococcosis in solid organ transplant recipients: clinical relevance of serum cryptococcal antigen. Clin Infect Dis. 2008;46(2):e12-e18. doi:10.1086/524738

[12]Setianingrum, Findra, Riina Rautemaa-Richardson, and David W. Denning. "Pulmonary cryptococcosis: a review of pathobiology and clinical aspects." Medical mycology 57.2 (2019): 133-150.

[13]Zavala, Sofia, and John W. Baddley. "Cryptococcosis." Seminars in respiratory and critical care medicine. Vol. 41. No. 01. Thieme Medical Publishers, 2020.

[14] Zhang PH, Hu BJ, He LX, et al. "The characteristics of CT imaging and diagnosis of pulmonary cryptococcosis in 42 cases with non-acquired immune deficiency syndrome" Chin J Intern Med. 2009;48(5):362-366.

[15] Qu Y, Liu G, Ghimire P, et al. Primary pulmonary cryptococcosis: evaluation of CT characteristics in 26 immunocompetent Chinese patients. Acta Radiol. 2012;53(6):668-674. doi:10.1258/ar.2012.110612

[16]Sui, Xin, et al. "Clinical features of pulmonary cryptococcosis in thin-section CT in immunocompetent and non-AIDS immunocompromised patients." La radiologia medica 125.1 (2020): 31-38. 
[17] Choi KH, Park SJ, Min KH, et al. Treatment of asymptomatic pulmonary cryptococcosis in immunocompetent hosts with oral fluconazole. Scand J Infect Dis. 2011;43(5):380-385. doi:10.3109/00365548.2011.552521

[18]PerfectJR, Dismukes WE, and F. Dromer. "Clinical practice guidelines for the management of cryptococcal disease: 2010 update by the infectious diseases society of America." ClinInfectDis 50.3 (2010): 291-322.

[19]Shi, Bingyi, et al. "Clinical diagnostic and treatment technique guidelines for invasive fungal disease in solid organ transplant recipients" Organ Trans ,2019,10(03):227-236.

[20] Lin, P C et al. "Dynamic research of serum cryptococcal capsular polysaccharide antigen titer and chest CT of pulmonary cryptococcosis after antifungal therapy" Natl Med J China vol. 99,26 (2019): 2036-2041. doi:10.3760/cma.j.issn.0376-2491.2019.26.007 